\title{
A novel recovery algorithm of time encoded signals
}

\author{
Dorian Florescu, Daniel Coca* \\ From Twenty Second Annual Computational Neuroscience Meeting: CNS*2013 \\ Paris, France. 13-18 July 2013
}

The integrate-and-fire (IAF) neuron model is a Time Encoding Machine (TEM), which maps analog signals into a sequence of strictly increasing time events. There are a number of reconstruction algorithms that ensure perfect recovery of bandlimited input signals from spike trains [1]. For signals that are not bandlimited, or when their bandwidth is unknown, the algorithms available [2] ensure that the reconstructed signal satisfies a consistency constraint, i.e., the reconstructed stimulus generates the same spike train as the original stimulus. As noted in [2], consistent reconstruction is more relevant and useful for recovering real sensory stimuli because a good estimate of the bandwidth is often not available. The existing algorithms that reconstruct the inputs encoded with TEMs exploit, in some form, the nonuniform sampling theory. Here we propose a novel consistent algorithm for signal reconstruction from spike trains, which involves solving an associated interpolation problem based on uniformly sampled data, and present theoretical results that underpin the proposed reconstruction method. While providing

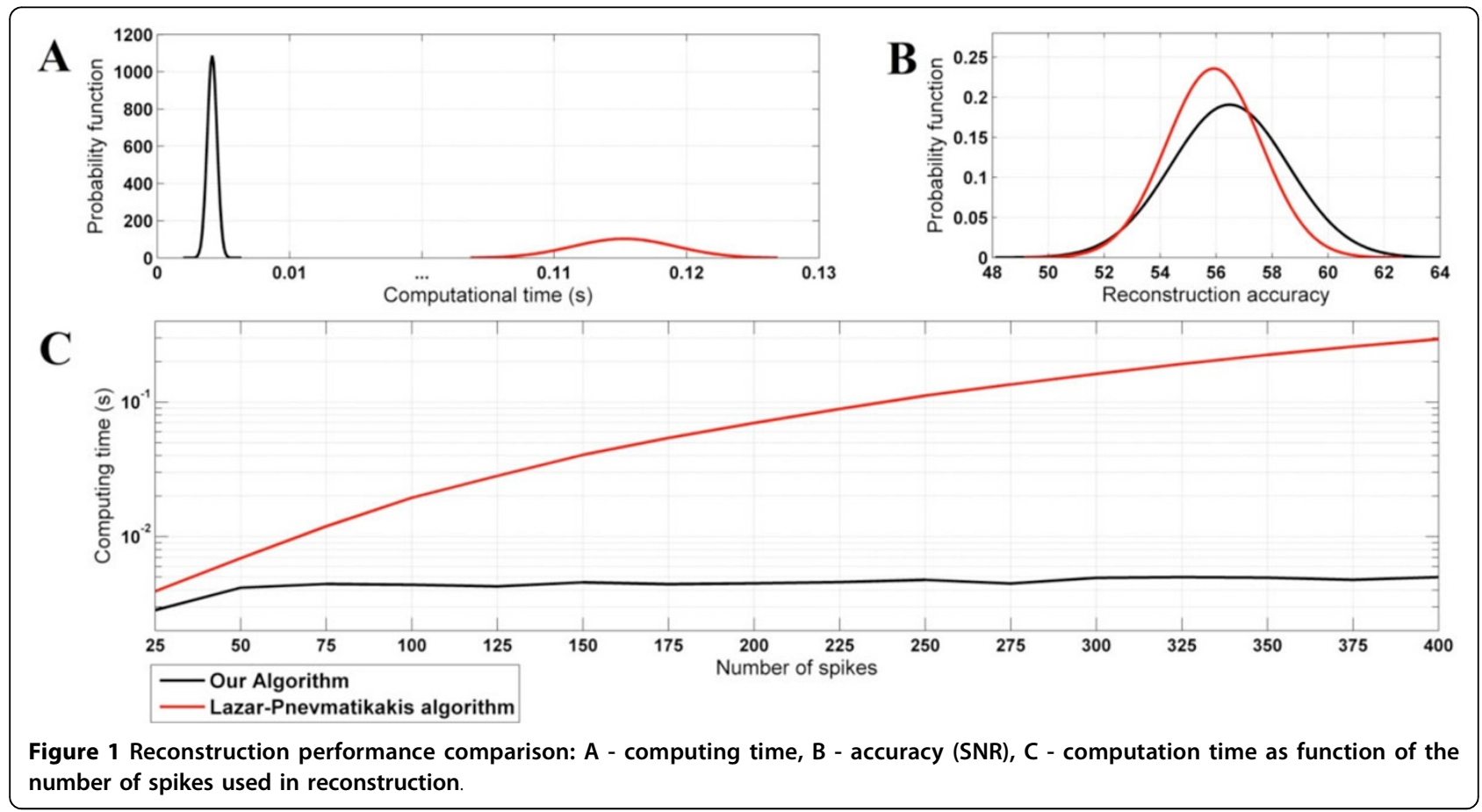

Department of Automatic Control and Systems Engineering, University of

Sheffield, Sheffield, South Yorkshire, S1 3JD, UK

() Biomed Central

(C) 2013 Florescu and Coca; licensee BioMed Central Ltd. This is an Open Access article distributed under the terms of the Creative Commons Attribution License (http://creativecommons.org/licenses/by/2.0), which permits unrestricted use, distribution, and reproduction in any medium, provided the original work is properly cited. 
similar accuracy, our algorithm is faster and thus better suited for real-time processing, because our approach does not require recalculating the basis functions used in reconstruction for different sets of spikes. In addition, the new approach provides an alternative framework to study spike processing. To show the performance of our algorithm, we compared it to the best consistent reconstruction method available [2]. The input used was a periodic signal with randomly generated Fourier coefficients. The computation time and reconstruction accuracy of each algorithm were evaluated for 100 randomly generated input sequences. The estimated probability density functions corresponding to each performance index are shown in Figures $1 \mathrm{~A} \& \mathrm{~B}$. The average computation time, plotted as a function of the number of spikes (Figure 1B), demonstrates that computing time is almost independent on the number of spikes. The simulations were carried out in Matlab on a $3.10 \mathrm{GHz}$ Intel Single Core PC workstation.

Published: 8 July 2013

\section{References}

1. Lazar AA: Time encoding with an integrate-and-fire neuron with a refractory period. Neurocomputing 2004, 58-60, 53-58.

2. Lazar AA, Pnevmatikakis EA: Consistent recovery of sensory stimuli encoded with MIMO neural circuits. Computational Intelligence and Neuroscience 2010, 2010, Article ID 469658.

doi:10.1186/1471-2202-14-S1-P130

Cite this article as: Florescu and Coca: A novel recovery algorithm of time encoded signals. BMC Neuroscience 2013 14(Suppl 1):P130.
Submit your next manuscript to BioMed Central and take full advantage of:

- Convenient online submission

- Thorough peer review

- No space constraints or color figure charges

- Immediate publication on acceptance

- Inclusion in PubMed, CAS, Scopus and Google Scholar

- Research which is freely available for redistribution

Submit your manuscript at www.biomedcentral.com/submit 